\title{
Infección del líquido pleural y ascítico por Listeria monocytogenes. Caso clínico
}

\author{
Raimundo López-Rodríguez, Joaquín Campos-Franco, \\ Nieves Mallo-González, María Rosario Alende-Sixto \\ Pleural and ascitic fluid infection \\ by Listeria monocytogenes. \\ Report of one case
}

Ascitic and pleural fluids infection by Listeria monocytogenes is uncommon. The association of spontaneous bacterial peritonitis and empyema caused by this microorganism has been seldom reported. A 61 year-old male with an alcoholic cirrhosis and an upper right lobectomy for a lung cancer, consulted because of an exacerbation of dyspnea, abdominal pain and fever. Listeria monocytogenes was isolated from ascitic and pleural fluids and from blood cultures. He was successfully treated with ampicillin and a chest tube for drainage (Rev Méd Chile 2008; 136: 1453-6).

(Key words: Ampicillin; Ascitic fluid; Listeria monocytogenes; Pleural effusion)

Recibido el 4 de enero, 2008. Aceptado el 13 de mayo, 2008.

Servicio de Medicina Interna, Complejo Hospitalario Universitario de Santiago de Compostela. Santiago de Compostela, A Coruña, España.

L a infección por Listeria monocytogenes, un bacilo Gram positivo aerobio y anaerobio facultativo, suele afectar a mujeres embarazadas, neonatos, personas de edad avanzada y sujetos inmunodeprimidos (neoplasias, trasplante de órgano sólido, infección por VIH, tratamiento con corticosteroides) ${ }^{1}$.

Las formas más frecuentes de presentación de la enfermedad son la meningitis y la bacteriemia ${ }^{1}$. En los últimos años se ha producido un incremento de los casos de peritonitis bacteriana espontánea (PBE) ocasionados por este microorganismo, recomendán-

Correspondencia a: Dr. Raimundo López Rodríguez. Servicio de Medicina Interna, Hospital Clínico Universitario c/ Travesía da Choupana s/n 15706, Santiago de Compostela, A Coruña. Tlfn: 981950522. Fax: 981950501.

E mail: lopezrodriguez.rm@gmail.com dose tenerlo presente en aquellos casos con mala evolución con el tratamiento empírico convencional, ya que la L monocytogenes es resistente a las cefalosporinas de tercera generación ${ }^{2-4}$. En la actualidad se han descrito 23 casos de infección del líquido pleural por Listeria monocytogenes, en los que sólo en tres se aisló también en el líquido ascítico ${ }^{4-12}$.

Presentamos un caso de infección del líquido pleural y ascítico por Listeria monocytogenes y realizamos una revisión de la literatura prestando especial atención a su epidemiología, patogenia, tratamiento y pronóstico.

\section{CASO CLÍNICO}

Varón de 61 años de edad con antecedentes de cirrosis hepática de etiología alcohólica, ascitis intratable con diuréticos que precisaba de para- 
centesis evacuadoras periódicas, hemorragia digestiva alta por várices esofágicas, lobectomía superior derecha por adenocarcinoma de pulmón hace 18 meses y diabetes mellitus tipo 2 a tratamiento con insulina. Consultó por aumento del perímetro abdominal y deterioro del estado general. En la exploración física destacaba una temperatura de $37,8^{\circ} \mathrm{C}$, presión arterial de $100 / 55$ $\mathrm{mmHg}$, frecuencia cardíaca de $98 \mathrm{lpm}$, taquipnea respirando aire ambiente, estigmas cutáneos de hepatopatía crónica, ictericia cutáneo mucosa, semiología de derrame pleural derecho y de ascitis.

En el estudio de laboratorio, que incluyó hemograma, perfil bioquímico y estudio de coagulación, se objetivaron 11.580 leucocitos (con $90 \%$ PMN, 3,1\% linfocitos, 5,2\% monocitos y $2 \%$ cayados), TQ $16 \mathrm{~s}, \mathrm{Hb} 11 \mathrm{~g} / \mathrm{dL}, \mathrm{VCM}$ 72,6 fl, plaquetas $126.000 / \mathrm{mm}^{3}$. Glucosa $139 \mathrm{mg} / \mathrm{dL}$, urea $44 \mathrm{mg} / \mathrm{dL}$ creatinina $0,8 \mathrm{mg} / \mathrm{dL}$, bilirrubina total $2,7 \mathrm{mg} / \mathrm{dL}$, albúmina $3,3 \mathrm{~g} / \mathrm{L}$, aspartato transaminasa (AST) $24 \mathrm{U} / \mathrm{L}$, alanina transaminasa (ALT) 30 $\mathrm{U} / \mathrm{L}$, gammaglutamiltransferasa (GGT) $108 \mathrm{U} / \mathrm{L}$, fosfatasa alcalina 358 UI/L, LDH 293 UI/L, VSG 52 $\mathrm{mm} \mathrm{1}$ a h. En la gasometría arterial presentaba $\mathrm{pH}$ 7,55, pO2 48,5 mmHg, pCO2 26,6 mmHg, bicarbonato $22,9 \mathrm{mEq} / \mathrm{L}$. La $\mathrm{Rx}$ tórax demostró la presencia de derrame pleural derecho y la ecografía abdominal un hígado pequeño con patrón ecográfico en vidrio deslustrado, tres nódulos en lóbulo derecho de 58, 32 y $27 \mathrm{~mm}$ compatibles con hepatocarcinomas, así como circulación colateral periesplénica, esplenomegalia y trombosis parcial de la vena porta.

$\mathrm{Al}$ ingreso se realizó una paracentesis evacuadora que obtuvo un líquido de aspecto turbio con 5.240 leucocitos/uL (80\% polimorfonucleares) y 0,6 $\mathrm{g}$ de proteínas /dL Ante estos hallazgos se inició tratamiento empírico con ceftriaxona intravenosa y albúmina (1,5 gr/ $\mathrm{Kg}$ de peso el primer día y $1 \mathrm{~g} / \mathrm{Kg}$ de peso el tercer día). A pesar de lo cual, el paciente no experimentó mejoría clínica, presentando deterioro de su estado general e incremento de la temperatura, por lo que se realizaron hemocultivos y una toracocentesis diagnóstica que mostró un pH 6,64, 12.000 leucocitos/uL (80\% polimorfonucleares), glucosa $1 \mathrm{mg} / \mathrm{dL}$, proteínas 1,6 g/L, LDH $309 \mathrm{UI} / \mathrm{L}$, colesterol $23 \mathrm{mg} / \mathrm{dL}$

La tinción de Gram evidenció la presencia de bacilos Gram positivos de aspecto corineforme y en los cultivos de sangre, líquido ascítico y pleural se aisló Listeria monocytogenes. Ante estos hallazgos se colocó un tubo de drenaje torácico y se sustituyó la cefalosporina de tercera generación por ampicilina $2 \mathrm{gr}$ iv/6 h. La evolución fue favorable y el paciente fue dado de alta a su domicilio al cumplir tres semanas de tratamiento antibiótico.

\section{DisCUSIÓN}

En los últimos años se ha puesto de manifiesto un aumento del número de casos de peritonitis bacteriana espontánea ocasionados por la Listeria monocytogenes, al menos 50 casos, y se ha remarcado la importancia de tenerla presente en aquellos casos de PBE que no evolucionen de forma favorable con tratamiento empírico habitual $^{2-4}$. Menos frecuentes son los casos de empiema por Listeria monocytogenes, así en el momento actual sólo se han notificado 23 casos en la literatura y únicamente en tres de ellos existía además infección del líquido ascítico ${ }^{4-12}$.

La infección del líquido pleural por este microorganismo se han producido inicialmente sobre todo en pacientes con neoplasias hematológicas $8,11,12$, sin embargo en los últimos tiempos se ha observado un incremento de su incidencia en personas con cirrosis hepática, como ocurre con el caso que presentamos ${ }^{4-7,9,10}$. Es de destacar que $13 \%$ de los casos se produjeron en personas sin enfermedad inmunosupresora, si bien es cierto que uno de ellos presentaba una fístula esofagopleural y los otros eran mayores de 60 años, lo que se considera una indicación para administrar antibióticos activos frente la L monocytogenes en el tratamiento empírico de las meningitis bacterianas.

$\mathrm{Si}$ en los pacientes con neoplasias hematológicas el incremento de la susceptibilidad para padecer una infección por L monocytogenes se explica porque sufren un deterioro de la inmunidad celular mediada por células $\mathrm{T}$, bien sea por la propia enfermedad o por el tratamiento con quimioterapia o corticoesteroides ${ }^{1}$. En los pacientes con cirrosis hepática se justifica porque presentan una disminución de la capacidad fagocítica del sistema reticuloendotelial (SRE) y de la actividad opsonizante del líquido ascítico, que muestra 
niveles bajos de proteínas y factores del complemento, se produce edema en la pared intestinal y una colonización bacteriana del intestino delgado. Además se objetiva una disminución del aclaramiento plasmático de bacterias por el SRE debido al desarrollo de colaterales portosistémicas y un incremento de los niveles sanguíneos de hierro, que in vitro favorece el crecimiento de la Listeria $3,7,9$.

La infección del líquido ascítico y pleural por Listeria monocytogenes sólo se ha notificado en cuatro ocasiones, incluido el caso que presentamos. El 50\% de los pacientes tenían una neoplasia hematológica, enfermedad de Hodgkin y linfoma no Hodgkin de células pequeñas, y el otro 50\% una enfermedad hepática, una cirrosis de etiología enólica y otra criptogenética. En los tres casos que se notifican los resultados de los hemocultivos, incluido nuestro paciente, éstos fueron positivos. Se trataron con penicilina, ampicilina, ceftriaxona y metronidazol y cotrimoxazol. Sólo se realizó drenaje evacuador en uno de los casos y la mortalidad fue de $50 \% 4,11,12$.

El tratamiento recomendado para la listeriosis en pacientes inmunodeprimidos es la ampicilina asociada a gentamicina ${ }^{1,12,13}$. Hay que tener en cuenta que en los pacientes cirróticos los aminoglucósidos debe emplearse con precaución, sobre todo si presentan insuficiencia renal, motivo por el cual nosotros no se los administramos a nuestro paciente. En los casos de alergia a la penicilina el cotrimoxazol es una alternativa eficaz ${ }^{13}$. El imipenen, el linezolid y las nuevas quinolonas, levofloxacino y moxifloxacino, han demostrado su actividad in vitro frente a la Listeria monocytogenes, pero aún está por determinar su papel en el tratamiento de la listeriosis ${ }^{14,15}$. La duración del tratamiento antibiótico no está claramente establecida y se recomiendan de tres a seis sema-

\section{REFERENCIAS}

1. LORBER B. Listeriosis. Clin Infect Dis 1997; 24: 1-11.

2. Espinoza-Gómez F, Newton-Sánchez O, Melnikov V, PInZón SL. Peritonitis bacteriana espontánea por Listeria monocytogenes, en un paciente con cirrosis hepática. Caso clínico. Rev Méd Chile 2006; 134: 1171-4.

3. Jammula P, Gupta R. Listeria monocytogenes- Induced nas 1,5,12,13. Tampoco está claramente establecida la indicación del tubo de drenaje torácico en los casos de empiema ${ }^{10}$. Tras revisar la casuística, incluido nuestro paciente, se objetiva que en 54\% de los casos en los que procedió a su colación la mortalidad fue de $30 \%$ y en los que no se utilizó fue de $36 \%$, sin embargo hay que tener en cuenta que cuando excluimos al paciente que no recibió ningún tratamiento, la mortalidad del grupo sin drenaje torácico desciende a $27 \%^{4-12}$. Ante estos hallazgos parece que una opción razonable puede ser iniciar el tratamiento antibiótico y en función de la evolución clínica, en las siguientes 48-72 h, decidir si es necesaria la indicación del drenaje torácico.

La mortalidad global de la infección por L monocytogenes, incluyendo el caso que presentamos, es de $33 \%$ en los pacientes con empiema y de $50 \%$ en aquellos que existe afectación del líquido pleural y ascítico. Tras revisar la literatura se objetiva que en los casos con empiema tienen un pronóstico mejor los pacientes con cirrosis hepática, mortalidad de $22 \%$, que los que sufren una neoplasia hematológica, mortalidad de 54\%412. Esta diferencia es todavía más marcada en los casos con infección del líquido pleural y ascítico, donde la mortalidad asciende a $100 \%$ en los pacientes con neoplasias hematológicas y no se produce en los que tenían enfermedad hepáti$\mathrm{ca}^{4,11,12}$.

En los últimos años se ha producido un incremento de los casos de infección del líquido ascítico o pleural por L monocytogenes en pacientes cirróticos, por lo que es importante tener presente este microorganismo en los pacientes cirróticos con PBE que no evolucionan de forma favorable con el tratamiento empírico habitual y en aquellos casos con derrame pleural y fiebre, ya que esta infección presenta un curso fulminante en los pacientes inmunodeprimidos.

monomicrobial non- neutrocytic bacterascites. South Med J 2002; 95: 1204-6.

4. Toyoshima MT, Apanavicius A, Soeiro AD, Almeida GM, ARAI MH. Listeria monocytogenes peritonitis in cirrhotic patients: first description in Brazil. Rev Inst Med Trop Sao Paulo 2006; 48: 291-3.

5. LuJAN RG, GarRido Vv, Quero CG. Derrame pleural por Listeria monocytogenes en mujer con cirrosis hepática. Arch Bronconeumol 2007; 43: 421. 
6. Janssens W, Van Raemdonck D, Dupont L, Verleden GM. Listeria pleuritis 1 week after lung transplantation. J Heart Lung Transplant 2006; 25: 734-7.

7. De Sa FR, Sztajnbok J, De Almeida JF, Troster EJ, Vaz FA. Listeria monocytogenes pneumonia in a cirrotic child. Int J Clin Pract 2004; 58: 536-8.

8. Tobalina Larrea I, López Legarra G, Martínez Odriozola P, Rojo P, Fresco Benito J, López Capel A et al. Derrame pleural por Listeria monocytogenes. Presentación de un caso y revisión de la literatura. An Med Interna 1999; 16: 463-5.

9. Unzaga Baranano MJ, Rojo de Ursua P, Pardo Moral C, Melero Almau P, Calvo Muro F et al. Infección del líquido pleural por Listeria monocytogenes. Enferm infecc Microbiol Clin 1997; 15: 337-8.

10. Marron A, Rosón B, Mascaró J, Carratalá J. Listeria monocytogenes empiema in an HIV infected patient. Thorax 1997; 52: 745-6.
11. Vay C, Meiero M, Mattera J, Famigletti A, Rigou RC. Serositis extensa por Listeria monocytogenes. Enf Infecc Microbiol Clin 1996; 14: 402.

12. MAZULi T, SALd IE. Pleural fluid infection caused by Listeria monocytogenes: case report and review. Rev Infect Dis 1991; 13: 564-70.

13. Jones EM, MacGowan AP. Antimicrobial chemotherapy of human infection due to Listeria monocytogenes. Eur J Clin Microbiol Infect Dis 1995; 14: 165-75.

14. Safdar A, Armstrong D. Antimicrobial activities against 84 Listeria monocytogenes isolates from patients with systemic listeriosis at a comprehensive cancer center (1955-1997). J Clin Microbiol 2003; 41: 483-5.

15. Jones RN, Biedenbach DJ, ANDEREgG TR. In vitro evaluation of AZD2563, a new oxazolidinone, tested against unusual gram-positive species. Diagn Microbiol Infect Dis 2002; 42: 119-22. 